\title{
III. Ventilación no invasiva en pacientes con enfermedades pulmonares obstructivas
}

\author{
FRANCISCO ARANCIBIA H.*, SEBASTIÁN UGARTE U.** y FERNANDO SALDÍAS P.***
}

\section{Noninvasive ventilation for acute exacerbations of obstructive pulmonary diseases}

Los principales objetivos de la ventilación no invasiva (VNI) son mejorar la oxigenación y la ventilación (corregir la acidosis respiratoria e hipercapnia) de los pacientes con insuficiencia respiratoria parcial o global, evitando la intubación y conexión a ventilador mecánico, reduciendo los riesgos y complicaciones asociados: lesiones de la vía aérea superior, neumonía nosocomial, sinusitis, prolongación de la estadía en UCI y el hospital ${ }^{1}$.

La ventilación mecánica no invasiva ha sido de utilidad en el tratamiento de algunas formas de insuficiencia respiratoria aguda y crónica, en particular en pacientes con enfermedad pulmonar obstructiva crónica (EPOC) $)^{2}$. En esta condición clínica se ha planteado la existencia de un estado de fatiga muscular respiratoria crónica que se explica por una excesiva carga mecánica secundaria a las altas resistencias al flujo aéreo y por hiperinsuflación toracopulmonar, que determina una relación longitud-tensión desventajosa de los músculos inspiratorios y hace que el trabajo muscular respiratorio sea menos eficiente ${ }^{3}$. Así, la VNI podría ser beneficiosa por varios aspectos. Aplicar una cantidad externa y apropiada de presión positiva continua en la vía aérea (CPAP) para contrarrestar la presión positiva al final de la espiración intrínseca (PEEPi) podría mejorar la mecánica respiratoria y reducir el trabajo muscular ${ }^{4}$. Por otro lado, valores crecientes de presión de soporte disminuyen el trabajo respiratorio y aumentan el volumen corriente y la ventilación minuto ${ }^{5}$.

En la exacerbación aguda de la EPOC, la CPAP mejora el intercambio gaseoso, hecho no demostrado de manera concluyente fuera de estos períodos de exacerbación ${ }^{4}$. La aplicación de CPAP también puede ser beneficiosa al contrarrestar la PEEPi, disminuyendo el trabajo respiratorio y el costo energético para el inicio de la respiración. La presión de soporte disminuye el trabajo de los músculos respiratorios, hecho de particular relevancia en pacientes con EPOC exacerbado $^{5}$. Cuando estos músculos están sometidos permanentemente a condiciones mecánicas desfavorables, experimentan un estado de fatiga muscular crónica que compromete su reserva funcional ${ }^{2}$. La presión de soporte reduce la actividad electromiográfica y el esfuerzo diafragmático tanto en etapa estable como en la agudización de la enfermedad. La VNI aumenta el volumen minuto y el volumen corriente, reduce la frecuencia respiratoria y el consumo de oxígeno de los músculos inspiratorios, y mejora el intercambio gaseoso en pacientes con EPOC exacerbado ${ }^{6}$.

\section{Ventilación no invasiva en pacientes con EPOC exacerbado}

Las exacerbaciones de la enfermedad pulmonar obstructiva crónica (EPOC) son motivo de consulta y hospitalización frecuentes, ocasionando incapacidad física prolongada, deterioro de la calidad de vida de los enfermos y gastos de salud elevados ${ }^{7}$. Tradicionalmente, los pacientes con EPOC tabáquica moderada-grave y exacerbación aguda que no respondían a la terapia tradicional (oxigenoterapia, broncodilatadores,

\footnotetext{
* Unidad de Cuidados Intensivos, Instituto Nacional del Tórax, Santiago, Chile.

** Presidente Sociedad Chilena de Medicina Intensiva, Jefe Unidad de Paciente Crítico, Hospital del Salvador y Clínica Indisa, Santiago.

*** Profesor Asociado de Medicina, Departamento de Enfermedades Respiratorias, Pontificia Universidad Católica de Chile.
} 
corticoides sistémicos) eran intubados y conectados a ventilación mecánica ${ }^{7}$. Esto, implicaba sedación e ingreso a una unidad de cuidados intensivos. Así mismo, la intubación y la VM están asociados a mayor morbilidad, riesgo de complicaciones y muerte ${ }^{8}$. La sobrevida media de los pacientes con EPOC exacerbado ventilados por falla respiratoria aguda bordea el $70 \%{ }^{7,8}$. Además, los pacientes con EPOC avanzado y reserva funcional respiratoria reducida pueden ser considerados una categoría especial, en quienes suele ser difícil el proceso de destete o desconexión del ventilador asociado a elevada morbimortalidad 9 .

Pregunta: ¿La ventilación no invasiva reduce el riesgo de intubación, complicaciones y muerte en pacientes con enfermedad pulmonar obstructiva crónica y falla respiratoria aguda?.

Pacientes: Pacientes hospitalizados por exacerbaciones de la enfermedad pulmonar obstructiva crónica en insuficiencia respiratoria aguda que necesitan de asistencia ventilatoria.

Intervención: VNI en pacientes con exacerbaciones graves de la EPOC.

Objetivo: Evaluar si la VNI reduce el riesgo de complicaciones, la mortalidad y la necesidad de VM invasiva en pacientes con EPOC exacerbado.

\section{Resumen de la evidencia}

En varios estudios realizados en la década del 90' se concluye que la VNI es una opción de tratamiento, alternativo a la ventilación mecánica invasiva, para pacientes hospitalizados con falla respiratoria hipercápnica secundaria a exacerbación aguda en pacientes portadores de EPOC $^{10-12}$. Brochard y Meduri, fueron los primeros en comunicar el uso de VNI por máscara facial en pacientes con EPOC y falla respiratoria aguda $^{6,10}$. El procedimiento fue bien tolerado y la respuesta fisiológica obtenida fue similar a la VM convencional. La VNI redujo la necesidad de intubación, la duración de la ventilación mecánica y la estadía en UCI. Desde entonces, la eficacia de la VNI en este grupo de pacientes ha sido evaluada extensamente. En la Tabla 1 se resumen los principales estudios clínicos que han examinado la eficacia de la VNI en pacientes con EPOC exacerbado.

Recientemente en una conferencia de consenso internacional, se recomendó que la VNI sea considerada como un tratamiento de primera línea en estos pacientes ${ }^{13}$. Esta recomendación está fundamentada en varios estudios clíni$\cos$ controlados $^{14,15}$. Brochard y cols ${ }^{14}$, estudiaron 85 pacientes con EPOC descompensado que ingresaron a UCI, los cuales fueron aleatorizados a tratamiento con VNI por máscara facial versus tratamiento estándar. El uso de VNI disminuyó significativamente la necesidad de intubación $(26 \%$ vs $74 \%, \mathrm{p}<0,001)$, el riesgo de complicaciones $(16 \%$ vs $48 \%, \mathrm{p}=0,001)$, la estadía en el hospital $(23 \pm 17$ días vs $35 \pm 33$ días, $\mathrm{p}=0,005)$ y la mortalidad hospitalaria ( $9 \%$ vs $29 \%, \mathrm{p}=0,02)$.

Plant y $\operatorname{cols}^{15}$, compararon la VNI y terapia estándar en 236 pacientes con EPOC que presentaron falla respiratoria aguda. Los pacientes fueron admitidos en salas de cuidados respiratorios generales, randomizados y en forma aleatoria se aplicó VNI o terapia estándar. La corrección de la acidosis respiratoria (aumento del $\mathrm{pH}$ arterial y disminución de la hipercapnia) y disminución de la frecuencia respiratoria fueron más rápidos y de mayor magnitud en el grupo que recibió apoyo ventilatorio. La tasa de intubación orotraqueal y conexión a ventilador mecánico $(15 \%$ vs $27 \%, \mathrm{p}=0,02)$, y la mortalidad intrahospitalaria $(10 \%$ vs $20 \%, \mathrm{p}=0,05)$ fueron menores en el grupo de VNI. Sin embargo, el beneficio en la mortalidad no era aparente en los pacientes más graves con $\mathrm{pH}$ arterial en la admisión al hospital menor de 7,30. Ambos estudios muestran resultados consistentemente favorables, con una tasa de éxito de la VNI entre el 75 y $85 \%$.

Ram y $\operatorname{cols}^{16}$, realizaron recientemente un meta-análisis cuyo objetivo fue determinar la eficacia de la VNI en el manejo de pacientes con falla respiratoria debido a una exacerbación aguda de la EPOC. En el análisis fueron incluidos 14 estudios controlados aleatorizados que evaluaron a 758 pacientes con EPOC exacerbado e insuficiencia respiratoria global ${ }^{11,12,14,15,17-26}$. El grupo que recibió VNI por máscara nasal o facial presentó disminución significativa de la frecuencia respiratoria y $\mathrm{PaCO}_{2}$, y aumento de la $\mathrm{PaO}_{2}$ una hora después de implementada la intervención comparado con el grupo que recibió terapia estándar. Los estudios demuestran una disminución significativa del riesgo de intubación (RR 0,41, IC95\% 0,33-0,53), riesgo de complicaciones (RR 0,38, IC95\% 0,24-0,60), estadía hospitalaria (diferencia media -3,24 días, IC95\% $-4,42$ a $-2,06$ ), y riesgo de muerte (RR 0,52, IC95\% 0,35-0,76). En un meta-análisis realizado por Keenan y cols, revelaron que los beneficios de la VNI se observaban sólo en los pacientes con exacerbaciones graves de la $\mathrm{EPOC}^{27}$. Estudios clínicos controlados y randomizados de buena calidad demuestran los beneficios de la VNI como terapia de primera línea en el manejo de la insuficiencia respiratoria aguda aso- 
ciada a exacerbaciones moderadas y graves de la EPOC. La Sociedad Americana de Tórax (ATS) y la Sociedad Británica de Tórax (BTS) recomiendan emplear la VNI en pacientes con EPOC exacerbado y acidosis respiratoria $(\mathrm{pH}$ $<7,35)$ que no responden con el tratamiento médico convencional ${ }^{13,28}$.

Tomados en conjunto, estos estudios proporcionan evidencia científica de que la VNI mejora el intercambio gaseoso o la oxigenación, corrige la acidosis respiratoria, disminuye el apremio respiratorio y acorta el tiempo de permanencia en la UCI y el hospital. Asimismo, esta intervención reduce la tasa de intubación, riesgo de complicaciones y muerte en el hospital, lo cual constituye el efecto de mayor trascendencia.

\section{Efecto potencial}

Los estudios clínicos randomizados muestran beneficios de la VNI como intervención de primera línea y terapia adyuvante adicionada al tratamiento médico habitual en pacientes con falla respiratoria aguda por exacerbación de la EPOC. La VNI disminuye la tasa de intubación, conexión a ventilador, riesgo de complicaciones y muerte en pacientes con EPOC exacerbado y falla respiratoria hipercápnica.

\section{Evaluación del costo/beneficio}

De acuerdo a la información disponible, es posible asegurar que la VNI en pacientes con EPOC exacerbado y falla respiratoria aguda hipercápnica es una medida costo/efectiva: disminuye el riesgo de intubación, conexión a ventilador y las complicaciones asociadas, acorta la estadía en UCI y el hospital, y reduce la mortalidad.

\section{Grado de recomendación}

La ventilación no invasiva debe ser considerada una terapia de primera línea en el manejo de la falla respiratoria aguda en pacientes con exacerbación moderada o grave de la EPOC (recomendación firme sustentada en evidencia de buena calidad).

\section{Ventilación no invasiva en pacientes con crisis asmática grave}

En los últimos años, la incidencia y gravedad del asma bronquial han ido en aumento, lo cual ha determinado un incremento significativo en el número de pacientes asmáticos manejados en unidades de cuidado crítico. El asma aguda grave se define como aquel "paciente asmático con crisis de broncoespasmo severo que no respon- de al tratamiento broncodilatador habitual, usado a dosis plena, y que desarrolla insuficiencia respiratoria aguda que pone en peligro la vida del enfermo"29. Los objetivos del tratamiento en estos casos en primer lugar es evitar la muerte del paciente y en forma secundaria pero no menos importante evitar la intubación y ventilación mecánica.

La fisiopatología de la falla respiratoria aguda en el paciente asmático tiene algunas similitudes con la falla respiratoria aguda de la exacerbación de la EPOC. Durante la crisis, los pacientes presentan aumento significativo de la obstrucción bronquial, trastornos V/Q e hiperinflación dinámica ${ }^{29}$. Los músculos inspiratorios se mantienen contraídos durante todo el ciclo respiratorio, y la sobredistensión torácica acorta la longitud de las fibras del diafragma y los músculos accesorios reduciendo su eficiencia mecánica. Finalmente, la fatiga de los músculos respiratorios y aumento del espacio muerto fisiológico ocasionan acidosis respiratoria y falla respiratoria. La intubación endotraqueal y conexión a ventilador mecánico se requiere en pacientes con crisis asmática grave refractaria al tratamiento convencional que desarrollan fatiga de los músculos respiratorios o complicaciones con riesgo vital (hipotensión, arritmias, confusión mental). Al igual que en la exacerbación aguda de la EPOC, parece razonable pensar que la VNI puede ser exitosa en el manejo del asma aguda grave ${ }^{30}$.

Varios estudios han demostrado los efectos beneficiosos del CPAP en el manejo de la crisis asmática grave ${ }^{29,31-34}$ : a) Disminuye la resistencia de la vía aérea y produce broncodilatación; b) Favorece la remoción de secreciones y la reexpansión de atelectasias; c) Disminuye el trabajo de los músculos respiratorios y contrarresta el auto-PEEP; d) Contrarresta los efectos deletéreos en la hemodinamia ocasionados por los cambios bruscos de presión negativa pleural.

Pregunta: ¿La VNI reduce el riesgo de complicaciones y muerte, y la necesidad de VM invasiva en pacientes con crisis asmática grave?.

Pacientes: Pacientes con crisis asmática grave y falla respiratoria aguda que necesitan de asistencia ventilatoria.

Intervención: VNI en pacientes con crisis asmática grave.

Objetivo: Evaluar si la VNI reduce el riesgo de complicaciones, la mortalidad y la necesidad de VM invasiva en pacientes con crisis asmática grave. 
Tabla 1. Estudios clínicos controlados que examinan la eficacia de la ventilación no invasiva en pacientes con EPOC exacerbado y falla respiratoria aguda

\begin{tabular}{|c|c|c|c|c|}
\hline Efecto de VNI & Bott $^{11}$ & Servillo $^{17}$ & Kramer $^{12}$ & Brochard $^{14}$ \\
\hline Publicación & 1993 & 1994 & 1995 & 1995 \\
\hline Modalidad & VNI-PS & VNI-PS & BiPAP & VNI-PS \\
\hline Interface & Máscara nasal & Máscara nasal & Máscara nasal & Máscara facial \\
\hline Pacientes & 60 pacientes con & 10 pacientes con & 23 pacientes con & 85 pacientes con \\
\hline & EPOC exacerbado & EPOC exacerbado & EPOC exacerbado & EPOC exacerbado \\
\hline Intervención & VNI: 30 Control: 30 & No reportado & VNI: 11 Control: 12 & VNI: 43 Control: 42 \\
\hline Intubación & $\begin{array}{l}\text { RR: 0,20 } \\
\text { (IC95\% 0,01-4,00) }\end{array}$ & $\begin{array}{l}\text { RR: 0,33 } \\
\text { (IC95\% 0,05-221) }\end{array}$ & $\begin{array}{l}\text { RR: } 0,14 \\
\text { (IC95\% 0,02-0,9) }\end{array}$ & $\begin{array}{l}\text { RR: } 0,35 \\
\text { (IC95\% 0,2-0,6) }\end{array}$ \\
\hline Fracaso de tratamiento & $\begin{array}{l}\text { RR: } 0,38 \\
\text { (IC95\% 0,16-0,94) }\end{array}$ & No reportado & No reportado & $\begin{array}{l}\text { RR: } 0,36 \\
\text { (IC95\% 0,2-0,6) }\end{array}$ \\
\hline Estadía en el hospital & $\begin{array}{l}\text { WMD: -1,40 } \\
\text { (IC95\% -5,08; 2,28) }\end{array}$ & No reportado & $\begin{array}{l}\text { WMD: }-2,40 \\
\text { (IC95\% }-10,7 ; 5,9)\end{array}$ & $\begin{array}{l}\text { WMD: }-12,00 \\
\text { (IC95\% }-23,2 ;-0,8)\end{array}$ \\
\hline Mortalidad & $\begin{array}{l}\text { RR: } 0,33 \\
\text { (IC95\% 0,10-1,11) }\end{array}$ & $\begin{array}{l}\text { RR: } 1,00 \\
\text { (IC95\% 0,08-11,9) }\end{array}$ & No reportado & $\begin{array}{l}\text { RR: 0,33 } \\
\text { (IC95\% 0,11-0,93) }\end{array}$ \\
\hline Efecto de VNI & Barbe $^{18}$ & Avdeev $^{20}$ & Celikel $^{19}$ & Plant $^{15}$ \\
\hline Publicación & 1996 & 1998 & 1998 & 2000 \\
\hline Modalidad & BiPAP & BiPAP & VNI-PS & VNI-PS \\
\hline Interface & Máscara nasal & Máscara nasal-facial & Máscara facial & Máscara nasal-facial \\
\hline Pacientes & 24 pacientes con & 58 pacientes con & 30 pacientes con & 236 pacientes con \\
\hline & EPOC exacerbado & EPOC exacerbado & EPOC exacerbado & EPOC exacerbado \\
\hline Intervención & VNI: 14 Control: 10 & VNI: 29 Control: 29 & VNI: 15 Control: 15 & VNI: 118 Control: 118 \\
\hline Intubación & No reportado & $\begin{array}{l}\text { RR: } 0,63 \\
\text { (IC95\% 0,23-1,6) }\end{array}$ & $\begin{array}{l}\text { RR: } 0,50 \\
\text { (IC95\% 0,05-4,9) }\end{array}$ & $\begin{array}{l}\text { RR: } 0,56 \\
\text { (IC95\% 0,3-0,9) }\end{array}$ \\
\hline Fracaso de tratamiento & $\begin{array}{l}\text { RR: 6,60 } \\
\text { (IC95\% 0,39-110) }\end{array}$ & $\begin{array}{l}\text { RR: 0,58 } \\
\text { (IC95\% 0,27-1,3) }\end{array}$ & $\begin{array}{l}\text { RR: } 0,17 \\
\text { (IC95\% 0,02-1,2) }\end{array}$ & $\begin{array}{l}\text { RR: } 0,63 \\
\text { (IC95\% 0,39-1,0) }\end{array}$ \\
\hline Estadía en el hospital & $\begin{array}{l}\text { WMD: }-0,70 \\
\text { (IC95\% }-3,84 ; 2,44 \text { ) }\end{array}$ & $\begin{array}{l}\text { WMD: }-8,00 \\
\text { (IC95\% }-12,4 ;-3,5)\end{array}$ & $\begin{array}{l}\text { WMD: }-2,90 \\
\text { (IC95\% }-5,8 ; 0,07 \text { ) }\end{array}$ & $\begin{array}{l}\text { WMD: }-0,62 \\
\text { (IC95\% }-4,48 ; 3,24 \text { ) }\end{array}$ \\
\hline Mortalidad & No reportado & $\begin{array}{l}\text { RR: } 0,33 \\
\text { (IC95\% 0,10-1,1) }\end{array}$ & $\begin{array}{l}\text { RR: } 0,33 \\
\text { (IC95\% 0,01-7,58) }\end{array}$ & $\begin{array}{l}\text { RR: } 0,50 \\
\text { (IC95\% 0,26-0,95) }\end{array}$ \\
\hline Efecto de VNI & Zhou $^{21}$ & Thys $^{24}$ & Khilnani $^{22}$ & Dikensoy $^{23}$ \\
\hline Publicación & 2001 & 2002 & 2002 & 2002 \\
\hline Modalidad & VNI-PS & BiPAP & BiPAP & BiPAP \\
\hline Interface & Máscara nasal & Máscara facial & Máscara nasal & Máscara facial \\
\hline Pacientes & $\begin{array}{l}60 \text { pacientes con } \\
\text { EPOC exacerbado }\end{array}$ & $\begin{array}{l}12 \text { pacientes con } \\
\text { EPOC exacerbado }\end{array}$ & $\begin{array}{l}40 \text { pacientes con } \\
\text { EPOC exacerbado }\end{array}$ & $\begin{array}{l}34 \text { pacientes con } \\
\text { EPOC exacerbado }\end{array}$ \\
\hline Intervención & VNI: 30 Control: 30 & VNI: 7 Control: 5 & VNI: 20 Control: 20 & VNI: 17 Control: 17 \\
\hline Intubación & $\begin{array}{l}\text { RR: 0,41 } \\
\text { (IC95\% 0,20-0,85) }\end{array}$ & $\begin{array}{l}\text { RR: 0,11 } \\
\text { (IC95\% 0,01-1,7) }\end{array}$ & $\begin{array}{l}\text { RR: 0,25 } \\
\text { (IC95\% 0,08-0,8) }\end{array}$ & $\begin{array}{l}\text { RR: 0,29 } \\
\text { (IC95\% 0,07-1,2) }\end{array}$ \\
\hline Fracaso de tratamiento & No reportado & $\begin{array}{l}\text { RR: } 0,07 \\
\text { (IC95\% 0,0-1,01) }\end{array}$ & No reportado & $\begin{array}{l}\text { RR: 0,51 } \\
\text { (IC95\% 0,18-1,45) }\end{array}$ \\
\hline Estadía en el hospital & No reportado & No reportado & No reportado & $\begin{array}{l}\text { WMD: }-4,30 \\
\text { (IC95\% }-6,16 ;-2,4)\end{array}$ \\
\hline Mortalidad & No reportado & No reportado & $\begin{array}{l}\text { RR: } 1,50 \\
\text { (IC95\% 0,28-8,04) }\end{array}$ & $\begin{array}{l}\text { RR: 0,50 } \\
\text { (IC95\% 0,05-5,01) }\end{array}$ \\
\hline Efecto de VNI & Conti $^{25}$ & Del Castillo ${ }^{26}$ & \multicolumn{2}{|l|}{$\operatorname{Ram}^{16}$} \\
\hline Publicación & 2002 & 2003 & \multicolumn{2}{|l|}{2004} \\
\hline Modalidad & VNI-PS & BiPAP & \multicolumn{2}{|l|}{ VNI-BiPAP } \\
\hline Interface & Máscara facial & Máscara nasal & \multicolumn{2}{|l|}{ Máscara nasal-facial } \\
\hline Pacientes & $\begin{array}{l}49 \text { pacientes con } \\
\text { EPOC exacerbado }\end{array}$ & $\begin{array}{l}41 \text { pacientes con } \\
\text { EPOC exacerbado }\end{array}$ & \multicolumn{2}{|l|}{758 pacientes con } \\
\hline Intervención & VNI: 23 VM: 26 & VNI: 20 Control: 21 & \multicolumn{2}{|l|}{ VNI: 378 Control: 380} \\
\hline Intubación & $\begin{array}{l}\text { RR: 0,52 } \\
\text { (IC95\% 0,35-0,77) }\end{array}$ & $\begin{array}{l}\text { RR: } 0,35 \\
\text { (IC95\% 0,04-3,1) }\end{array}$ & \multicolumn{2}{|l|}{$\begin{array}{l}\text { RR: } 0,41 \\
(\text { IC95\% } 0.33-0.53)\end{array}$} \\
\hline Fracaso de tratamiento & No reportado & No reportado & \multicolumn{2}{|l|}{$\begin{array}{l}\text { RR: } 0,48 \\
\text { (IC95\% 0,37-0.63) }\end{array}$} \\
\hline Estadía en el hospital & No reportado & No reportado & \multicolumn{2}{|l|}{$\begin{array}{l}\text { WMD: }-3,24 \\
\text { (IC95\% }-4,42 ;-2,1 \text { ) }\end{array}$} \\
\hline Mortalidad & $\begin{array}{l}\text { RR: } 1,36 \\
\text { (IC95\% 0,48-3,86) }\end{array}$ & No reportado & \multicolumn{2}{|l|}{$\begin{array}{l}\text { RR: 0,52 } \\
\text { (IC95\% 0,35-0,76) }\end{array}$} \\
\hline
\end{tabular}

Nota: EPOC: enfermedad pulmonar obstructiva crónica, VNI-PS: presión de soporte-PEEP, VM: ventilación mecánica, RR: riesgo relativo, IC: intervalo de confianza, WMD: diferencia de la media (días). 
Tabla 2. Eficacia de la ventilación no invasiva en pacientes con crisis asmática severa

\begin{tabular}{|c|c|c|c|c|}
\hline Efecto de VNI & Martin $^{31}$ & Meduri $^{35}$ & Fernández ${ }^{36}$ & Soroksky $^{38}$ \\
\hline Publicación & 1982 & 1996 & 2001 & 2003 \\
\hline Modalidad & CPAP & VNI-PS & VNI-CPAP & BiPAP \\
\hline Interface & Máscara nasal & Máscara facial & Máscara facial & Máscara nasal \\
\hline Pacientes & $\begin{array}{l}8 \text { pacientes con } \\
\text { crisis asmática } \\
\text { inducida por } \\
\text { histamina }\end{array}$ & $\begin{array}{l}17 \text { pacientes con } \\
\text { crisis asmática } \\
\text { grave }\end{array}$ & $\begin{array}{l}22 \text { pacientes con } \\
\text { crisis asmática } \\
\text { grave }\end{array}$ & $\begin{array}{l}30 \text { pacientes con } \\
\text { crisis asmática } \\
\text { grave }\end{array}$ \\
\hline Intervención & CPAP: 8 & VNI: 17 & VNI: 22 VM: 11 & BiPAP: 15 Control: 15 \\
\hline Tipo de estudio & Experimental & Observacional & Observacional & Controlado \\
\hline Fracaso de VNI & No corresponde & $12 \%$ & $14 \%$ & $20 \%$ \\
\hline Efecto de VNI & $\begin{array}{l}\text { El CPAP reduce } \\
\text { la carga de los MI, } \\
\text { y mejora su } \\
\text { eficiencia }\end{array}$ & $\begin{array}{l}\text { Corrige la acidosis } \\
\text { respiratoria, } \\
\text { disminuye la FR, } \\
\text { y mejora oxigenación }\end{array}$ & $\begin{array}{l}\text { La estadía en UCI } \\
\text { y el hospital y } \\
\text { letalidad fueron } \\
\text { similares }\end{array}$ & $\begin{array}{l}\text { Disminuye la FR, } \\
\text { obstrucción bronquial } \\
\text { y la tasa de } \\
\text { hospitalización }\end{array}$ \\
\hline
\end{tabular}

Nota: CPAP: presión positiva continua en la vía aérea, VNI-PS: presión de soporte-PEEP, VM: ventilación mecánica, MI: músculos inspiratorios, FR: frecuencia respiratoria.

\section{Resumen de la evidencia}

Existen a lo menos tres estudios no controlados en paciente con crisis de asma bronquial severa y VNI que muestran resultados promisorios (Tabla 2). En un estudio experimental, Martin y cols ${ }^{31}$, en el año 1982 inducen broncoespasmo de intensidad moderada en 8 pacientes asmáticos mediante nebulización con histamina y luego les aplican CPAP $12 \mathrm{~cm}_{2} \mathrm{O}$. Ellos reportan una mejoría del volumen corriente, volumen minuto y flujo espiratorio. La aplicación de CPAP disminuyó la resistencia de la vía aérea y la sobrecarga de trabajo de los músculos inspiratorios, mejorando su eficiencia y el costo de la respiración. Meduri y cols ${ }^{35}$, en el año 1996 reporta la utilización de VNI en 17 pacientes con crisis asmática grave e insuficiencia respiratoria aguda, con un $\mathrm{pH}$ arterial promedio de 7,25. Ellos aplicaron VNI con éxito en 15 pacientes y sólo 2 casos requirieron intubación y ventilación mecánica invasiva. La VNI disminuyó el apremio respiratorio y la frecuencia respiratoria, corrigió la acidosis respiratoria y mejoró la oxigenación a las 2 horas. En otro estudio retrospectivo, Fernández y cols ${ }^{36}$, emplearon VNI en 22 pacientes con asma grave, el procedimiento fue bien tolerado por 19 pacientes, sólo tres pacientes fueron intubados y conectados a $\mathrm{VM}$, de los cuales uno falleció. Los autores concluyen que la VNI por máscara facial permite mejorar la ventilación alveolar y puede reducir la necesidad de intubación en grupos seleccionados de pacientes con crisis asmática grave.

Recientemente, fue publicado un análisis de la literatura de los estudios randomizados controlados en pacientes con crisis de asma grave y que se utilizó $\mathrm{VNI}^{37}$. Los autores encontraron sólo un estudio realizado por Soroksky y $\operatorname{cols}^{38}$, en Israel en una unidad de emergencias. Se incluyeron pacientes con asma bronquial que cumplían cuatro criterios de gravedad: a) $\mathrm{VEF}_{1}<$ $60 \%$ del predicho; b) frecuencia respiratoria mayor de $30 \mathrm{resp} / \mathrm{min}$; c) historia de asma durante más de un año, y d) duración del ataque de asma menor de 7 días. Se evaluaron 33 pacientes con asma aguda que recibieron terapia convencional y en forma randomizada se aplicó a 17 pacientes ventilación no invasiva durante tres horas con aumento gradual de las presiones hasta alcanzar un IPAP de $15 \mathrm{~cm} \mathrm{H}_{2} \mathrm{O}$ y EPAP de 5 cm $\mathrm{H}_{2} \mathrm{O}$, comparado con 16 pacientes a los cuales se les aplicó un IPAP y EPAP de $1 \mathrm{~cm}$ $\mathrm{H}_{2} \mathrm{O}$. Los autores demuestran que el uso de VNI puede mejorar la función respiratoria (aumento del $\mathrm{VEF}_{1}$ de $53,5 \pm 23,4 \%$ en el grupo con VNI vs $28,5 \pm 22,6 \%$ en el grupo control, $\mathrm{p}=0,006$ ), disminuir la frecuencia respiratoria y la intensidad del ataque rápidamente, y reducir significativamente la necesidad de hospitalización (VNI: 17,6\% vs CT: 62,5\%; RR: 0,28; IC95\% 0,09$0,84)$. En ambos grupos, no se reportaron fallecimientos ni necesidad de intubación y conexión a ventilador mecánico.

\section{Efecto potencial}

La aplicación de VNI en pacientes con crisis asmática grave o estatus asmático puede ser beneficiosa en algunos pacientes con falla respi- 
ratoria aguda hipoxémica, los estudios controlados aún son escasos y se requiere mayor evidencia para sustentar una recomendación.

\section{Evaluación del costo/beneficio}

De acuerdo a la información disponible, no es posible asegurar que la VNI en pacientes con crisis asmática grave y falla respiratoria aguda sea costo/efectiva. Se necesita efectuar estudios multicéntricos randomizados para aclarar este aspecto.

\section{Grado de recomendación}

La ventilación no invasiva puede ser una terapia de primera línea en pacientes con crisis asmática grave y falla respiratoria aguda hipoxémica (recomendación débil sustentada en evidencia de baja calidad). No existe suficiente evidencia en la literatura científica para recomendar el uso rutinario de VNI en pacientes con estatus asmático.

\section{Bibliografía}

1.- MEHTA S, HILL N S. Noninvasive ventilation. Am J Respir Crit Care Med 2001; 163: 540-77.

2.- DÍAZ O, BÉGIN P, TORREALBA B, JOVER E, LISBOA C. Effects of noninvasive ventilation on lung hyperinflation in stable hypercapnic COPD. Eur Respir J 2002; 20: 1490-8.

3.- BELMAN M J, SOO HOO G W, KUEI J H, SHADMEHR R. Efficacy of positive vs. negative pressure ventilation in unloading the respiratory muscles. Chest 1990; 98: 850-6.

4.- DE LUCAS P, TARANCÓN C, PUENTE L, RODRIGUEZ C, TATAY E, MONTURIOL J M. Nasal continuous positive airway pressure in patients with COPD in acute respiratory failure. A study of the immediate effects. Chest 1993; 104: 1694-7.

5.- APPENDINI L, PATESSIO A, ZANABONI S, CARONE M, GUKOV B, DONNER C F, et al. Physiologic effects of positive end-expiratory pressure and mask pressure support during exacerbations of chronic obstructive pulmonary disease. Am J Respir Crit Care Med 1994; 149: 1069-76.

6.- BROCHARD L, ISABEY D, PIQUET J, AMARO P, MANCEBO J, MESSADI A A, et al. Reversal of acute exacerbations of chronic obstructive lung disease by inspiratory assistance with a face mask. N Engl J Med 1990; 323: 1523-30.

7.- WEDZICHA J A, SEEMUNGAL T A. COPD exacerbations: defining their cause and prevention. Lancet 2007; 370: 786-96.

8.- UCGUN I, METINTAS M, MORAL H, ALATAS F, YILDIRIM H, ERGINEL S. Predictors of hospital outcome and intubation in COPD patients admitted to the respiratory ICU for acute hypercapnic respiratory failure. Respir Med 2006; 100: 66-74.

9.- MEYER T J, HILL N S. Noninvasive positive pressure ventilation to treat respiratory failure. Ann Intern Med 1994; 120: 760-70.
10.- MEDURI G U, ABOU-SHALA N, FOX R C, JONES C B, LEEPER K V, WUNDERINK R G. Noninvasive face mask mechanical ventilation in patients with acute hypercapnic respiratory failure. Chest 1991; 100: 44554.

11.- BOTT J, CARROLL M P, CONWAY J H, KEILTY S E, WARD E M, BROWN A M, et al. Randomised controlled trial of nasal ventilation in acute ventilatory failure due to chronic obstructive airways disease. Lancet 1993; 341: 1555-7.

12.- KRAMER N, MEYER T J, MEHARG J, CECE R D, HILL N S. Randomized, prospective trial of noninvasive positive pressure ventilation in acute respiratory failure. Am J Respir Crit Care Med 1995; 151: 1799806.

13.- International Consensus Conferences in Intensive Care Medicine: noninvasive positive pressure ventilation in acute respiratory failure. Am J Respir Crit Care Med 2001; 163: 283-91.

14.- BROCHARD L, MANCEBO J, WYSOCKI M, LOFASO F, CONTI G, RAUSS A, et al. Noninvasive ventilation for acute exacerbations of chronic obstructive pulmonary disease. N Engl J Med 1995; 333: 817-22.

15.- PLANT P K, OWEN J L, ELLIOTT M W. Early use of non-invasive ventilation for acute exacerbations of chronic obstructive pulmonary disease on general respiratory wards: a multicentre randomised controlled trial. Lancet 2000; 355: 1931-5.

16.- RAM F S, PICOT J, LIGHTOWLER J, WEDZICHA J A. Non-invasive positive pressure ventilation for treatment of respiratory failure due to exacerbations of chronic obstructive pulmonary disease. Cochrane Database Syst Rev 2004; 3: CD004104.

17.- SERVILLO G, UGHI L, ROSSANO F, LEONE D. Non invasive mask pressure support ventilation in COPD patients. Intensive Care Med 1994; 20 (suppl): S54.

18.- BARBÉ F, TOGORES B, RUBI M, PONS S, MAIMÓ A, AGUSTI A G. Noninvasive ventilatory support does not facilitate recovery from acute respiratory failure in chronic obstructive pulmonary disease. Eur Respir J 1996; 9: 1240-5.

19.- CELIKEL T, SUNGUR M, CEYHAN B, KARAKURT S. Comparison of noninvasive positive pressure ventilation with standard medical therapy in hypercapnic acute respiratory failure. Chest 1998; 114: 1636-42.

20.- AVDEEV S N, TRET'IAKOV A V, GRIGOR'IANTS R A, KUTSENKO M A, CHUCHALIN A G. Study of the use of noninvasive ventilation of the lungs in acute respiratory insufficiency due exacerbation of chronic obstructive pulmonary disease. Anesteziol Reanimatol 1998; 3: 45-51.

21.- ZHOU R, CHEN P, LUO H, XIANG X D. Effects of noninvasive positive pressure ventilation on gas exchange and patients' transformation in chronic obstructive pulmonary disease and respiratory failure. Hunan Yi Ke Da Xue Xue Bao 2001; 26: 261-2.

22.- KHILNANI G C, SAIKIA N, SHARMA S K, PANDE J $\mathrm{N}$, MALHOTRA O P. Efficacy of non-invasive positive pressure ventilation (NPPV) for management of COPD with acute or acute on chronic respiratory failure: a randomised controlled trial. ATS Annual Conference, 2002.

23.- DIKENSOY O, IKIDAG B, FILIZ A, BAYRAM N. Comparison of non-invasive ventilation and standard medical therapy in acute hypercapnic respiratory failure: a randomised controlled study at a tertiary health centre in SE Turkey. Int J Clin Pract 2002; 56: 85-8. 
24.- THYS F, ROESELER J, REYNAERT M, LIISTRO G, RODENSTEIN D O. Noninvasive ventilation for acute respiratory failure: a prospective randomised placebocontrolled trial. Eur Resp J 2002; 20: 545-55.

25.- CONTI G, ANTONELLI M, NAVALESI P, ROCCO M, BUFI M, SPADETTA G, et al. Noninvasive vs. conventional mechanical ventilation in patients with chronic obstructive pulmonary disease after failure of medical treatment in the ward: a randomized trial. Intensive Care Med 2002; 28: 1701-7.

26.- DEL CASTILLO D, BARROT E, LASERNA E, OTERO R, CAYUELA A, CASTILLO GÓMEZ J. Noninvasive positive pressure ventilation for acute respiratory failure in chronic obstructive pulmonary disease in a general respiratory ward. Med Clin (Barc) 2003; 120: 647-51.

27.- KEENAN S P, SINUFF T, COOK D J, HILL N S. Which patients with acute exacerbation of chronic obstructive pulmonary disease benefit from noninvasive positive-pressure ventilation? A systematic review of the literature. Ann Intern Med 2003; 138: 861-70.

28.- British Thoracic Society Standards of Care Committee. Non-invasive ventilation in acute respiratory failure. Thorax 2002; 57: 192-211.

29.- RODRIGO G J, RODRIGO C, HALL J B. Acute asthma in adults: a review. Chest 2004; 125: 1081-102.

30.- GEHLBACH B, KRESS J P, KAHN J, DERUITER C, POHLMAN A, HALL J. Correlates of prolonged hospitalization in inner-city ICU patients receiving noninvasive and invasive positive pressure ventilation for status asthmaticus. Chest 2002; 122: 1709-14.
31.- MARTIN J G, SHORE S, ENGEL L A. Effect of continuous positive airway pressure on respiratory mechanics and pattern of breathing in induced asthma. Am Rev Respir Dis 1982; 126: 812-7.

32.- ANDERSEN J B, QVIST J, KANN T. Recruiting collapsed lung through collateral channels with positive end-expiratory pressure. Scand J Respir Dis 1979; 60: 260-6.

33.- SHIVARAM U, DONATH J, KHAN F A, JULIANO J. Effects of continuous positive airway pressure in acute asthma. Respiration 1987; 52: 157-62.

34.- JARDIN F, DUBOURG O, MARGAIRAZ A, BOURDARIAS J P. Inspiratory impairment in right ventricular performance during acute asthma. Chest 1987; 92: 789-95.

35.- MEDURI G U, COOK T R, TURNER R E, COHEN $\mathrm{M}$, LEEPER K V. Noninvasive positive pressure ventilation in status asthmaticus. Chest 1996; 110: 767-74.

36.- FERNÁNDEZ M M, VILLAGRA A, BLANCH L, FERNÁNDEZ R. Non-invasive mechanical ventilation in status asthmaticus. Intensive Care Med 2001; 27: 486-92.

37.- RAM F S, WELLINGTON S, ROWE B, WEDZICHA $\mathrm{J}$ A. Non-invasive positive pressure ventilation for treatment of respiratory failure due to severe acute exacerbations of asthma. Cochrane Database Syst Rev 2005; 3: CD004360.

38.- SOROKSKY A, STAV D, SHPIRER I. A pilot prospective, randomized, placebo-controlled trial of bilevel positive airway pressure in acute asthmatic attack. Chest 2003; 123: 1018-25.

Correspondencia a:

Dr. Francisco Arancibia H.

E-mail: franciscoarancibia@hotmail.com 